\title{
Globular cluster abundances: the imprint of first-generation massive stars
}

\author{
Corinne Charbonnel \\ Geneva Observatory, University of Geneva, 51 Chemin des Maillettes, 1290 Versoix, \\ Switzerland \\ email: Corinne.Charbonnel@unige.ch \\ Laboratoire d'Astrophysique de Toulouse-Tarbes, Université de Toulouse, CNRS, France
}

\begin{abstract}
Galactic globular cluster (GC) stars exhibit abundance patterns that are not shared by their field counterparts, namely the well-documented $\mathrm{O}-\mathrm{Na}, \mathrm{C}-\mathrm{N}$ and $\mathrm{Mg}-\mathrm{Al}$ anticorrelations. Recent observations provide compelling evidence that these abundance anomalies were already present in the intracluster gas from which the presently observed stars formed. The current explanation is that the gas was polluted very early in the history of the GC by material processed through $\mathrm{H}$ burning at high temperatures and then lost by stars more massive than the longlived stars we still observe today. However the 'polluters' have not yet been unambiguously identified. Most studies have focused on asymptotic giant brach stars, but rotating massive stars present an interesting alternative. Here, we critically analyse the pros and cons of both potential stellar polluters. We discuss the constraints that the observational data provide on stellar nucleosynthesis and hydrodynamics, as well as on the formation and early evolution of very massive star clusters.
\end{abstract}

Keywords. stars: abundances, globular clusters: general

\section{Chemical dissection of globular clusters}

The classical paradigm describing globular clusters (GC) as fairly simple, large aggregates of coeval stars formed from homogeneous chemical material has been seriously challenged recently. There is indeed well-established evidence that GCs actually host multiple stellar populations. Much of this evidence comes from an increasing number of photometric observations in Galactic GCs but also in Magellanic Cloud clusters. Multiple populations manifest themselves in a split of different evolutionary sequences as observed in the cluster colour-magnitude diagrams.

The credit of this discovery, however, certainly goes to stellar spectroscopists, who have been collecting an incredible amount of data over the last three decades, revealing the striking chemical properties of GCs. At the beginning, only the brightest, most evolved stars were 'reachable'. A major step forward was achieved with the advent of 8-10m-class telescopes, which enabled access to abundance analysis in GC turnoff stars.

We refer to Gratton et al. (2004), Sneden (2005), and Decressin (2007) for extensive reviews of observed abundance trends in GCs. We briefly recall here the main points. (i) Individual GCs appear to be fairly homogeneous as far as Fe (Carretta et al. 2009a) and the iron-peak elements $(\mathrm{Ni}, \mathrm{Cu})$ are concerned (with the notable exception of $\omega$ Cen). (ii) They exhibit very low scatter and the same trends as field stars for the neutron-capture elements (both s- and r-process elements; Ba, La, Eu) and the $\alpha$ elements (Si, Ca). (iii) They exhibit, however, specific patterns and large star-to-star abundance variations in the lighter elements from $\mathrm{C}$ to $\mathrm{Al}$, which are not shared by their field counterparts. Let us focus on these light-element 'anomalies'. 
After the pioneering work of Osborn (1971), several studies showed that GCs are very heterogeneous in $\mathrm{C}$ and $\mathrm{N}$, and that anticorrelated variations in molecular-band strengths of $\mathrm{CN}$ and $\mathrm{CH}$ exist all the way down to the main-sequence turnoff in all GCs where data are available. Star-to-star variations of the strengths of the $\mathrm{Na}$ and $\mathrm{Al}$ lines at the tip of the red-giant branch (RGB) were discovered at the end of the 1970s (Cohen 1978; Peterson 1980; Norris et al. 1981). An anti-correlation between $\mathrm{O}$ and $\mathrm{Na}$ abundances (see Pilachowski 1989; Drake et al. 1992; Sneden et al. 1991 for the earliest studies) exists among evolved turnoff and RGB stars in all GCs studied to date (Carretta et al. 2009b). Whenever all necessary data are available, $\mathrm{N}$ is found to be anticorrelated with $\mathrm{O}$, which itself positively correlates with $\mathrm{C}$, and the sum $[(\mathrm{C}+\mathrm{N}+\mathrm{O}) / \mathrm{Fe}]$ appears to be constant within the observational errors (Decressin et al. 2009a; and references therein), with the possible exception of NGC 1851 (Yong et al. 2009). In addition, variations in $\mathrm{Al}$ and $\mathrm{Mg}$, again correlated with each other, have been discovered (e.g., Shetrone 1996; Ivans et al. 1999; Ramirez \& Cohen 2002; Carretta et al. 2009b). The sum $\mathrm{Mg}+\mathrm{Al}$ is also found to be constant in several clusters (Shetrone 2006). Last but not least, observations in NGC 6752, M 13, and M 71 show that a large spread in the ratio ${ }^{24} \mathrm{Mg}:{ }^{25} \mathrm{Mg}:{ }^{26} \mathrm{Mg}$ exists, combined with a positive correlation between ${ }^{26} \mathrm{Mg}$ and $\mathrm{Al}$ as well as an anticorrelation between ${ }^{24} \mathrm{Mg}$ and $\mathrm{Al}$, but no correlation between ${ }^{25} \mathrm{Mg}$ and $\mathrm{Al}$ (Yong et al. 2003, 2005, 2006). Additionally, the Li abundance was found to be anticorrelated with that of $\mathrm{Na}$ in turnoff stars in a couple of GCs (Pasquini et al. 2005; Bonifacio et al. 2007; Lind et al. 2009).

As an illustration, we show in Figure 1 the abundance patterns in NGC 6752, which is (to date) one of the best-studied GCs in terms of its chemical properties. The largest $\mathrm{O}$ values are similar to those of field-halo stars of the same metallicity as NGC 6752 $([\mathrm{Fe} / \mathrm{H}]=-1.5 \mathrm{dex})$. We can thus assume that this is the original $\mathrm{O}$ abundance of the intracluster gas. This is also true for the corresponding values of $\mathrm{Na}, \mathrm{Al}$, and $\mathrm{Mg}$. We can consider then that all abundances corresponding to the highest $\mathrm{O}$ abundances (as indicated by open circles in Figure 1) are the initial values and that abundances at the other extreme (indicated by open squares) correspond to the most extremely processed material. For the initial abundances of $\mathrm{C}$ and $\mathrm{N}$, we have to adopt $[\mathrm{C} / \mathrm{N}]$ and $[\mathrm{N} / \mathrm{Fe}]=0$, in view of the corresponding abundances of field-halo stars (Spite et al. 2005). We note that the corresponding initial abundances for Mg isotopes, based on observations of NGC 6752 do not correspond exactly to the scarce available observations of field-halo stars of the same metallicity. Pasquini et al. (2005) reported observations of Li in nine turnoff stars of NGC 6752 with UVES and found that $\mathrm{Li}$ is correlated with $\mathrm{O}$ and anticorrelated with Na. Stars with 'original' O (high) and $\mathrm{Na}$ (low) have a Li content similar to field-halo stars in the Spite plateau, i.e., $A(\mathrm{Li})=\log (\mathrm{Li} / \mathrm{H})+12 \sim 2.3$ dex. Stars with 'extreme' abundances (lowest $\mathrm{O}$ and highest $\mathrm{Na}$ ) are not devoid of $\mathrm{Li}$, but have a significant amount of $A(\mathrm{Li}) \sim 2$ dex.

Very importantly, high-resolution spectrographs on very large telescopes allow us to determine the abundances of the relevant elements in faint subgiant and turnoff stars in several GCs. The same chemical patterns are found independently of the evolutionary status of the stars, which is crucial, as discussed in Section 2. All these features are considered anomalous because they are not seen in field stars of similar metallicity (e.g., Gratton et al. 2000). However they could be considered 'universal' as far as GCs are concerned, since they have been found in all GCs where they have been looked for so far. Consequently, at a conference in Santa Barbara in January 2009, Piotto proposed a new definition for GCs that we should certainly adopt in future textbooks: "GCs are large aggregates of stars showing the O-Na anticorrelation". 
O b servations of NGC 6752
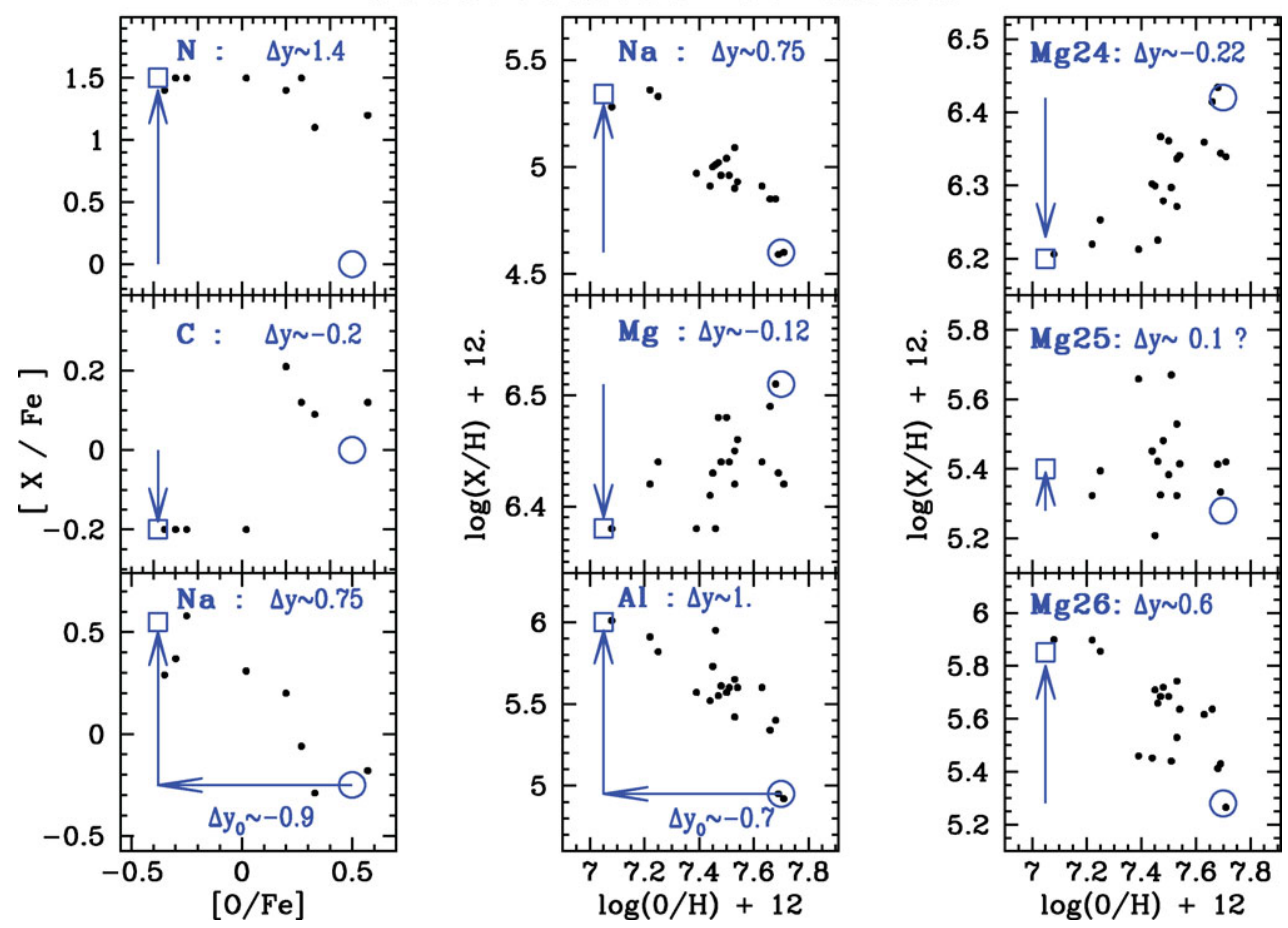

Figure 1. Abundances of $\mathrm{C}, \mathrm{N}, \mathrm{Na}, \mathrm{Mg}, \mathrm{Al}$ and $\mathrm{Mg}$ isotopes in NGC 6752 stars as a function of the corresponding oxygen abundance. Observations (filled symbols) in the left column (plotted as a function of $[\mathrm{O} / \mathrm{Fe}]$ ) are from Carretta et al. (2005) for turnoff stars, and in the other two columns (plotted as a function of $\mathrm{O} / \mathrm{H}$ ) from Yong et al. (2003) for bright giant stars. In all figures, the estimated initial abundances of the gas from which the cluster was formed are indicated by an open circle (i.e., it is assumed that they have the same composition as field-halo stars of the same metallicity- $[\mathrm{Fe} / \mathrm{H}]=-1.5$ dex-with, e.g., $[\mathrm{O} / \mathrm{Fe}]=0.5,[\mathrm{C} / \mathrm{Fe}]$ $=0$, etc.). The most extreme abundances observed at present in NGC 6752 are indicated by open squares. Arrows indicate the magnitude and direction of the abundance spread, which is also given by $\Delta y$ [in dex, where $y=\log$ (abundance)] in each panel. $\Delta y_{0}$ in the bottom panels indicates the corresponding variation in the oxygen abundance, larger in the case of Carretta et al. (2005; a factor of $\sim 8$ ) than in Yong et al. (2003; a factor of 5). In the former case, the authors have tried to enhance the chance of including extreme cases by selecting stars with likely strong and weak CN bands using the Strömgren $c_{1}$ index. (Figure from Prantzos et al. 2007: PCI07.)

\section{The self-enrichment scenario and the potential polluters}

The observed abundance patterns are the signatures of the simultaneous operation of the CNO, NeNa and MgAl cycles of hydrogen burning (Denissenkov \& Denissenkova 1990; Langer et al. 1993; Langer \& Hoffman 1995). However, although the CNO cycle is activated for temperatures on the order of $20 \times 10^{6} \mathrm{~K}$, the $\mathrm{NeNa}$ and $\mathrm{MgAl}$ chains require temperatures around $35 \times 10^{6} \mathrm{~K}$ and $50 \times 10^{6} \mathrm{~K}$, respectively. Destruction of ${ }^{24} \mathrm{Mg}$ by proton capture needs still higher temperatures, around $70 \times 10^{6} \mathrm{~K}$ (e.g., Arnould et al. 1999). Prantzos et al. (2007; hereafter PCI07) actually showed that the observed patterns of all elements and isotopes in a typical GC like NGC 6752 require $\mathrm{H}$ burning in a narrow range of temperatures around $75 \times 10^{6} \mathrm{~K}$. This is much higher than the internal temperature of the low-mass turnoff stars that we currently observe (see Figure 3). As a 


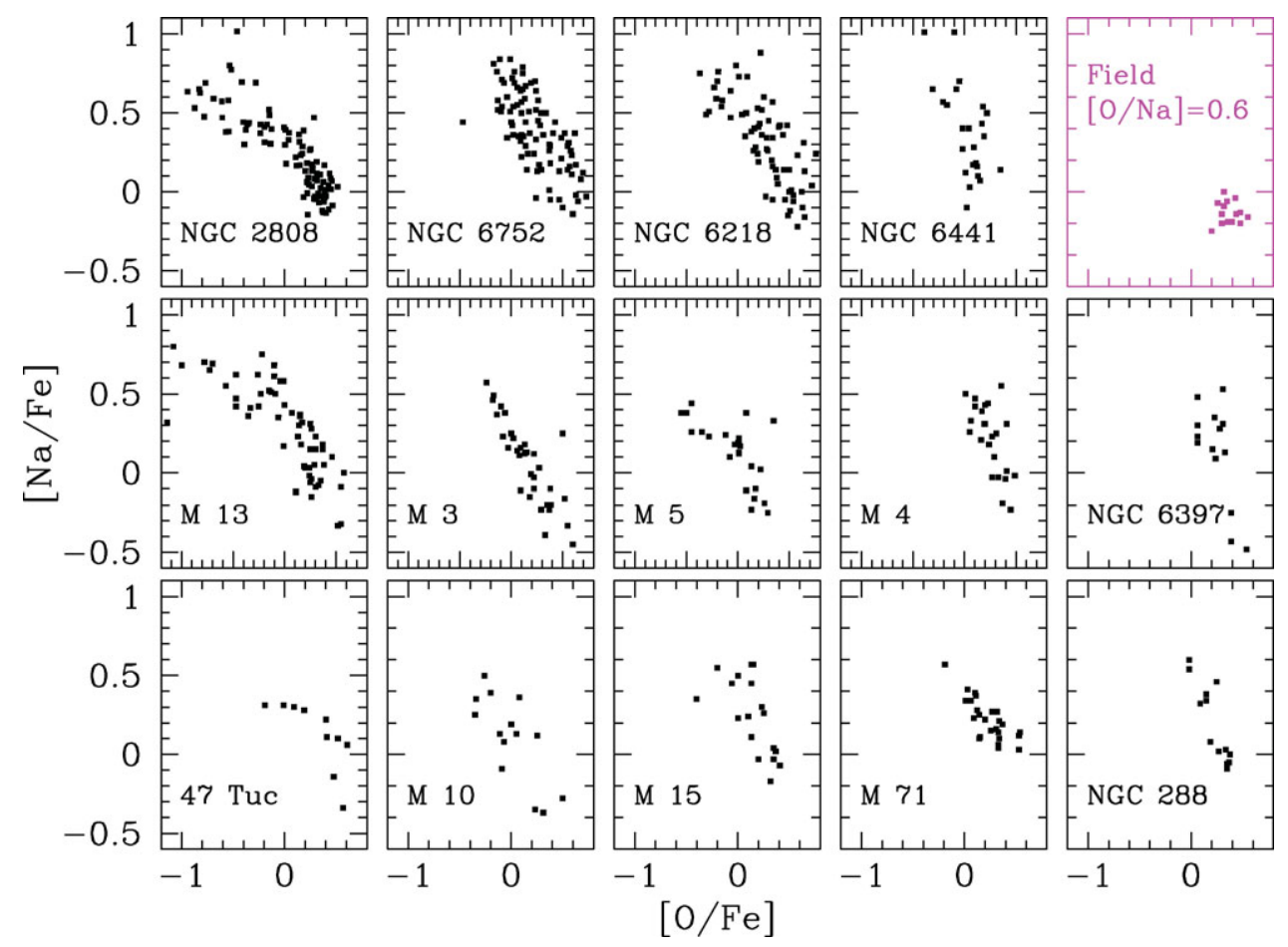

Figure 2. O-Na anticorrelation observed in several Galactic GCs and in field stars (for references, see Decressin 2007).

consequence, the abundance variations cannot be produced in situ, but certainly reflect the initial composition of the protostars. It is thus clear now that a large fraction of low-mass GC stars were formed from material processed through $\mathrm{H}$ burning at high temperatures and then lost by more massive and faster evolving stars, and partially mixed with some original gas.

In other words, compelling evidence leads us to believe that at least two generations of stars evolved in all Galactic GCs during their infancy. The first corresponds to the 'bulk' of normal stars born with the pristine composition of the protocluster gas. These objects are those with the highest $\mathrm{O}$ and $\mathrm{Mg}$ and the lowest $\mathrm{Na}$ and $\mathrm{Al}$ abundances also found in their field contemporaries. The second generation consists of stars formed from material polluted to various degrees by the ejecta of more massive and short-lived stars, and which present lower $\mathrm{O}$ and $\mathrm{Mg}$ and higher $\mathrm{Na}$ and $\mathrm{Al}$ abundances than their first-generation counterparts. Various aspects of this 'self-enrichment scenario' are developed in Prantzos \& Charbonnel (2006, hereafter PC06) and Decressin et al. (2007a,b).

Because we have very strong nucleosynthesis constraints, we can get clues as to the potential polluters by looking at the temperature at which hydrogen burning occurs in various types of stars. Figure 3 shows the maximum temperature reached in the $\mathrm{H}-$ burning regions for stars of various initial masses at different stages of their evolution. The horizontal dotted lines delimit the temperature range of $\mathrm{H}$ burning within the progenitors of the chemical anomalies, constrained by the combination of the observational data for $\mathrm{Na}, \mathrm{Al}$ and the $\mathrm{Mg}$ isotopes (see PCI07 for more details). One sees that there are actually two potential polluters: massive asymptotic giant branch (AGB) stars and massive stars. 


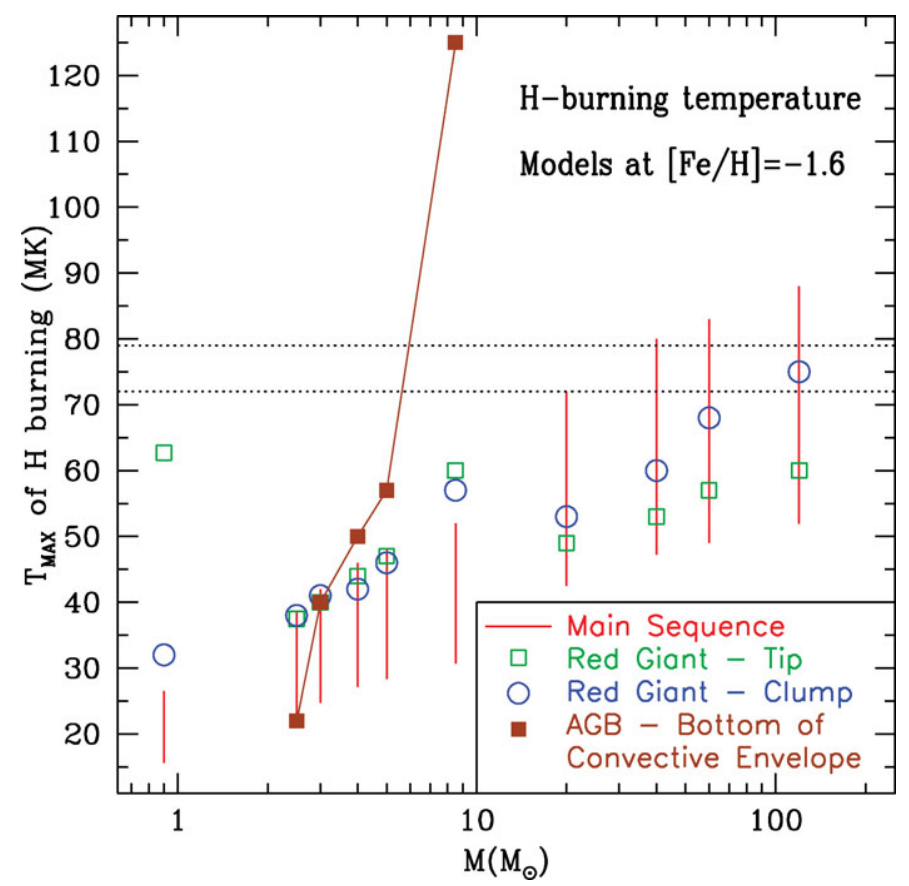

Figure 3. H-burning temperatures at various phases of a star's life as a function of initial stellar mass. The correspondence between symbols and evolutionary phases is displayed in the bottom right part of the figure. Vertical straight lines (for central H burning) start at the zero-age main sequence and end at central $\mathrm{H}$ exhaustion. For the other phases we show only the maximum temperature reached in the H-burning zones of interest. The two horizontal dotted lines enclose the temperature range reproducing the extreme abundances that are observed in NGC 6752 . (Figure from PCI07.)

\section{Were AGB stars the polluting agents?}

Massive AGB stars that undergo efficient hot-bottom burning (HBB) during the thermal-pulse phase (TP-AGB) have been proposed as possible GC polluters in this socalled self-enrichment scenario (Ventura et al. 2001). The AGB hypothesis has been discussed extensively in the literature, first on a qualitative basis, and more recently with the help of custom-made standard (i.e., nonrotating) stellar models (see references in Decressin et al. 2009a). These studies have pointed out several difficulties in generating the observed chemical patterns in theoretical TP-AGB models. The main problem is due to the competition between the third dredge up (3DUP) which contaminates the AGB envelope with the helium-burning ashes produced in the thermal pulse and HBB which modifies the envelope abundances through the $\mathrm{CNO}$ cycle and the $\mathrm{NeNa}$ and $\mathrm{MgAl}$ chains. It is thus very difficult to obtain simultaneous $\mathrm{O}$ depletion and $\mathrm{Na}$ enrichment in the $\mathrm{TP}-\mathrm{AGB}$ envelope, while keeping the $\mathrm{C}+\mathrm{N}+\mathrm{O}$ sum constant as required by the observations (for more details and references, see Charbonnel 2007).

To date, only massive (i.e., 5-6.5 $\mathrm{M}_{\odot}$ ) AGB models manage to achieve simultaneously an encouraging agreement with the observed $\mathrm{O}$ depletion and $\mathrm{Na}$ enrichment (Ventura \& D'Antona 2008a). These models include the 'full spectrum of turbulence' (FST) formulation for convection that strongly affects the $\mathrm{O}$ depletion once the stars enter the TP-AGB (Ventura \& D'Antona 2005a). Compared to the classical mixing-length theory treatment, FST leads indeed to higher temperatures at the base of the convective envelope (resulting 
in more advanced nucleosynthesis) and induces higher surface luminosities, resulting in stronger mass loss and thus fewer thermal pulses and 3DUP events.

The level of $\mathrm{Na}$ enrichment is more difficult to estimate and requires fine tuning of the NeNa-cycle reaction rates. More precisely, an increase in sodium is achieved in the most oxygen-poor ejecta of the $5-6.5 \mathrm{M}_{\odot}$ models only when the maximum allowed values for the ${ }^{22} \mathrm{Ne}(\mathrm{p}, \gamma)$ rate are adopted. In summary, and as clearly stated by Ventura \& D'Antona (2008a), the AGB scenario is viable from a nucleosynthesis point of view, provided that only massive AGB stars of 5 to $6.5 \mathrm{M}_{\odot}$ contribute to the GC self-enrichment, and under the physical assumptions described above. This causes severe difficulties in terms of mass budget and initial mass function (IMF) of the first stellar generation (see PC06).

The previously quoted studies have focused only on physical uncertainties related to the TP-AGB phase, and their predictions have not been tested in different astrophysical contexts. In particular, the impact of rotation on the nucleosynthesis predictions for AGB stars had never been investigated in the context of the GC self-enrichment scenario, although rotation is often invoked to understand a wide variety of observations (see, e.g., Maeder \& Meynet 2000, 2006; Chiappini et al. 2008). Decressin et al. (2009a) addressed this question for the first time. Using up-to-date treatment for rotation-induced processes, they investigated the effects of rotation up to the completion of the second dredge up (2DUP) and arrival on the AGB.

During central helium burning, rotational mixing efficiently transports primary ${ }^{12} \mathrm{C}$ and ${ }^{16} \mathrm{O}$ outside the convective core in the H-burning region, where these elements are processed by the CNO cycle, resulting in important production of primary ${ }^{14} \mathrm{~N}$. In the massive (initial mass greater or equal to $\sim 4 \mathrm{M}_{\odot}$ ) rotating models, after central He exhaustion the convective envelope penetrates into the layers affected by rotation-induced mixing. In contrast to standard models, the 2DUP then produces significant surface enrichment in total $\mathrm{C}+\mathrm{N}+\mathrm{O}$ that cannot be erased by hot-bottom (hydrogen) burning during the subsequent $\mathrm{TP}-\mathrm{AGB}$ evolution. Third dredge-up episodes can only further increase the total $\mathrm{C}+\mathrm{N}+\mathrm{O}$ mass fraction, as they bring to the surface the products of He burning. As a consequence, if rotating massive AGB stars were responsible for the abundance patterns observed in GCs, one would expect large $\mathrm{C}+\mathrm{N}+\mathrm{O}$ differences between (O-rich and Na-poor) first-generation stars and (O-poor and Na-rich) secondgeneration stars, as shown in Figure 4.

This behaviour is in sharp contrast with what is observed in low- and intermediatemetallicity GCs. To date, indeed, no significant star-to-star variation of the total $\mathrm{C}+\mathrm{N}+\mathrm{O}$ has been detected, except in NGC 1851 (Yong et al. 2009). We note, however, that the stars observed by Yong and collaborators in NGC 1851 are very bright objects. In view of its position in the colour-magnitude diagram, the only star with $\mathrm{C}+\mathrm{N}+\mathrm{O}$ exceeding the typical error bar could actually be an AGB star. In that case, the high value of the total CNO may not have been inherited at birth by the star, but may be due to nuclear processes within the star itself. Its possible nature as an AGB star would also explain why this star is the only object in the sample to exhibit some enhancement in s-process elements. CNO measurements in NGC 1851 of unevolved stars (as done in most of the other clusters studied so far) are thus crucial to settle the issue.

Our (Decressin et al. 2009a) study leads us to the conclusion that massive, rotating AGB stars can be discarded as potential polluters in the self-enrichment scenario in GCs, unless the crowded environment prevented intermediate-mass stars from rotating. This latest hypothesis is highly improbable in view of observations finding higher stellar rotation velocities in clusters than in the field (see Decressin et al. 2009a; and references therein). 


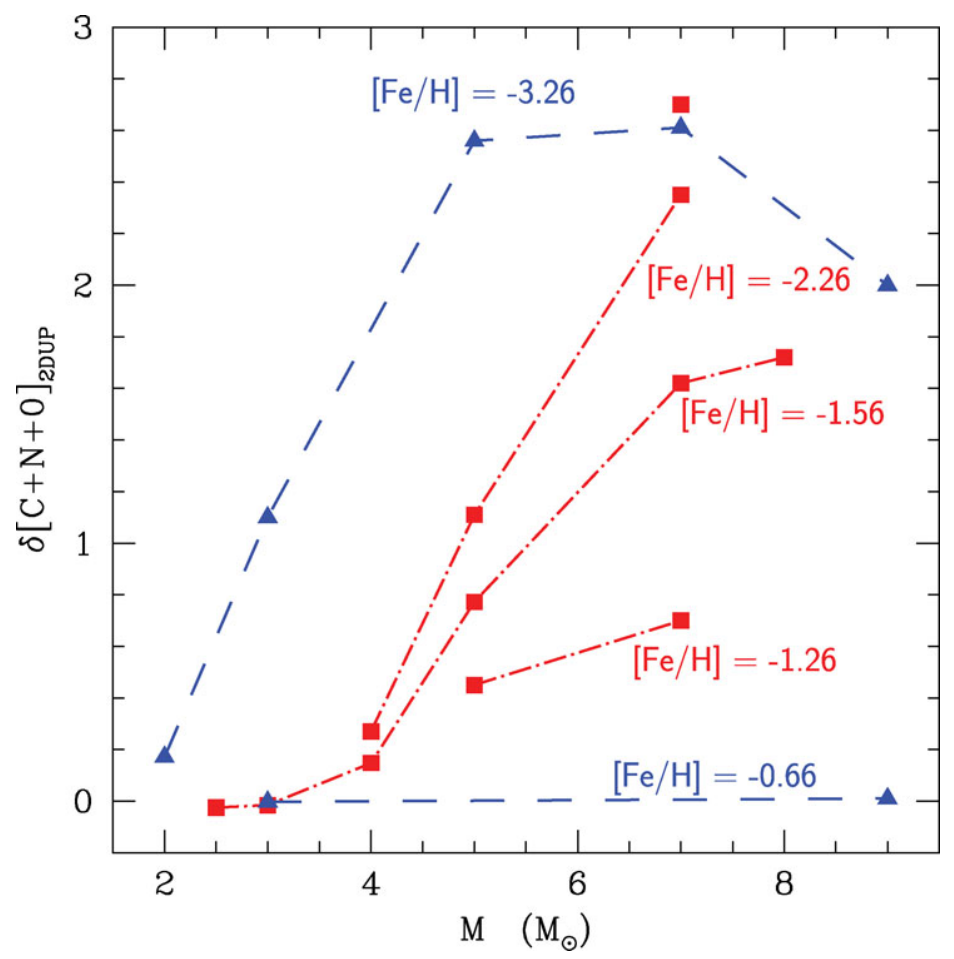

Figure 4. Surface $\mathrm{C}+\mathrm{N}+\mathrm{O}$ increase at the end of the 2DUP for rotating stars with various initial masses and metallicities. Squares and triangles indicate models computed with, respectively, STAREVOL and the Geneva code.

PC06 discuss other shortcomings of the AGB scenario, the main one being related to the peculiar IMF it requires. In addition, they underline the fact that the AGB scenario gives no satisfactory answer as to the role of stars more and less massive than the presumed polluters.

\section{The alternative: the wind of fast-rotating massive stars (WFRMS) scenario}

The idea that the winds of massive stars (hereafter WMS) may be at the origin of helium anomalies in GC stars was suggested by Norris (2004) and Maeder \& Meynet (2006) to explain the blue main sequence of the cluster $\omega$ Cen: the high helium content of the stars on that sequence could originate from WMS which produce a large helium/metal ratio. The 'wind of fast-rotating massive stars' (WFRMS) scenario was then proposed by PC06 and further developed by Decressin et al. (2007a,b; hereafter DMCPE07 and DCM07) in the more general context of the GC abundance anomalies described in Section 1.

As shown in Figure 3, the central temperatures of main-sequence massive stars (i.e., with an initial mass higher than $\sim 25 \mathrm{M}_{\odot}$ ) reach the high values required to produce the observed abundance anomalies. In DMCPE07 we computed models of rotating massive stars with the Geneva code, using different prescriptions for the nuclear reaction rates. In the core of main-sequence massive stars, the temperature is such that the CNO cycle is in equilibrium. There, the $\mathrm{C}-\mathrm{N}$ and $\mathrm{O}-\mathrm{Na}$ anticorrelations are easily generated, although the extent of $\mathrm{Na}$ production depends on the adopted reaction rates for the $\mathrm{NeNa}$ chain. In 
addition, $\mathrm{Al}$ is produced. However, $\mathrm{Mg}$ is also produced, unless one changes significantly the rate of the ${ }^{24} \mathrm{Mg}(\mathrm{p}, \gamma)$ reaction with respect to the nominal value published by Illiadis et al. (2001). In that case, the $\mathrm{Mg}-\mathrm{Al}$ anticorrelation builds up in the stellar core. At the same time, the central abundances of ${ }^{24} \mathrm{Mg}$ and ${ }^{26} \mathrm{Mg}$, respectively, decrease and increase, while ${ }^{25} \mathrm{Mg}$ remains almost constant. These theoretical patterns correspond to those observed in GC stars.

In DMCPE07 we suggested that fast rotation on the main sequence allows massive stars to eject the hydrogen-burning products through slow winds that can be easily retained within the GC potential well. Fast rotation velocities have undeniably a very important impact on mass loss. Indeed, when a star rotates at the break-up velocity (by this, we mean that the equatorial surface velocity is such that the centrifugal acceleration balances gravity), it loses a very important fraction of its mass through a mechanical wind. This mechanically ejected matter has a very low velocity (on the order of $20 \mathrm{~km} \mathrm{~s}^{-1}$ or less) and very likely forms an equatorial disk around the star, similar to that observed around Be-type stars (Ekström et al. 2008; see Figure 5).

Fast rotation not only drastically changes (qualitatively and quantitatively) stellar mass loss. It also modifies, through meridional circulation and shear turbulence that it induces within the star, the stellar chemical structure as well as the composition at the stellar surface and within the wind, as shown in Figure 5. As a consequence, the surfaceabundance variations during the main sequence mimic the central values, but with some delay. Within the conditions described above (see, in particular, the assumption on the ${ }^{24} \mathrm{Mg}(\mathrm{p}, \gamma)$ rate), the surface and wind composition of rotating massive stars present the H-burning signatures observed in GC stars.

The main reason as to why massive stars have been discarded in the past is related to the fact that they are expected to also produce heavier elements. Of course, within our framework, one has to avoid further contamination by the products of He burning and by the yields in heavy elements of Type II supernovae. Regarding the former, DMCPE07 note that once a star leaves the main sequence its rotation velocity suddenly decreases. Consequently, the wind velocities strongly increase and the corresponding ejected matter is probably lost from the GC. On the other hand, if black holes form through core collapse, they could swallow most of the pre-supernova yields (i.e., most of the heavy elements formed after the main-sequence phase). More probably, supernova ejection resulting from an initialy fast-rotating star, if it occurs, may favor ejection along jets aligned along the rotational axis. Of course, these ideas are highly speculative and need to be checked through detailed hydrodynamical simulations.

\section{Dynamical issues}

The schematic evolution of a GC is sketched in Figure 6. Based on the determination of the composition of giant stars in NGC 2808 by Carretta et al. (2006), PC06 determined that around $70 \%$ of stars exhibit abundance anomalies in this specific cluster. Decressin et al. (2007a) find similar results for NGC 6752 in their analysis of the data of Carretta et al. (2007): around $85 \%$ of the cluster stars (in a sample of 120 stars) show abundance anomalies. Therefore, most stars still evolving in globular clusters seem to be secondgeneration stars.

How can we produce such a high fraction of chemically peculiar stars? The main problem is that assuming a Salpeter (1955) IMF for the polluters, the accumulated mass of the slow winds ejected by the fast-rotating massive stars would only provide $10 \%$ of the total number of low-mass stars. To match the observations thus requires either (a) a flat IMF with a slope of 0.55 instead of the canonical Salpeter slope of 1.35 or (b) that $95 \%$ of the first-generation stars have escaped the cluster (Decressin et al. 2007a). 

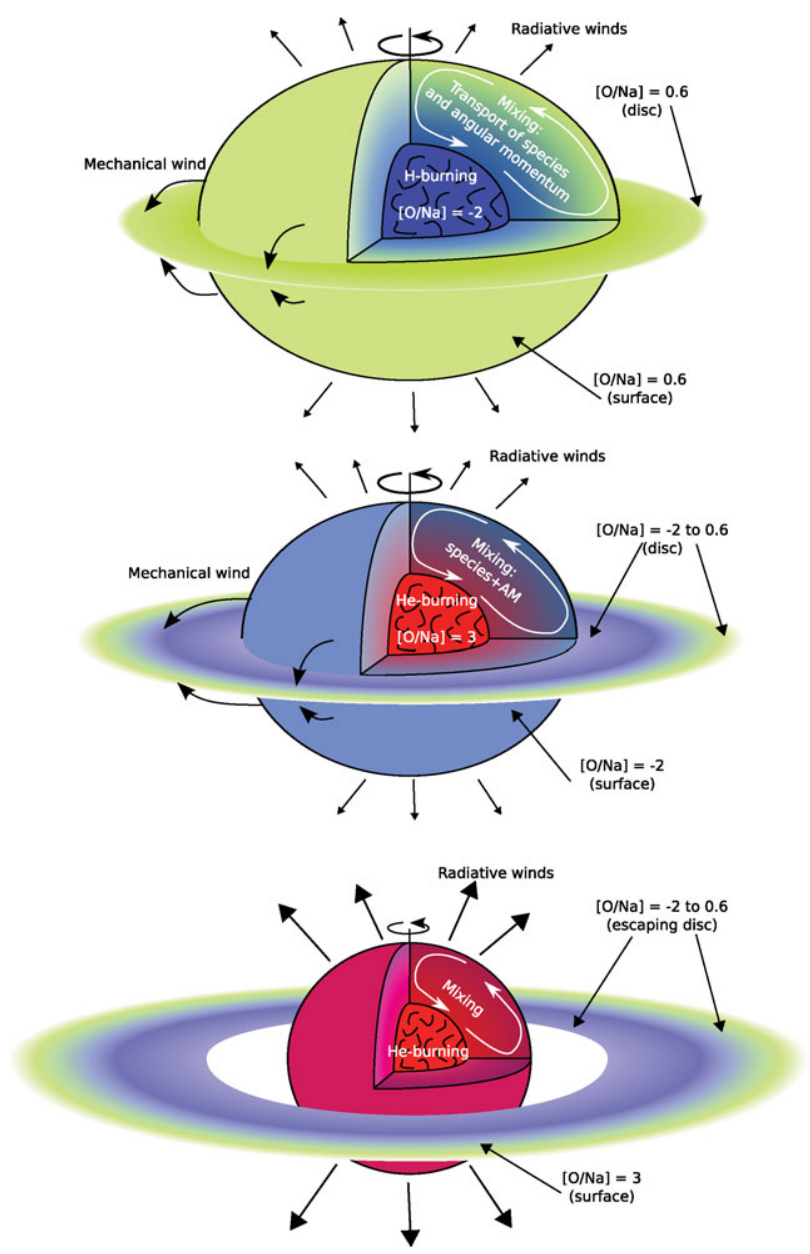

Figure 5. Schematic view of the evolution of fast-rotating massive stars. The colours reflect the chemical composition of the various stellar regions and of the disk: green corresponds to the initial chemical composition, blue and red are, respectively, for material loaded in $\mathrm{H}$ - and He-burning products. The typical $[\mathrm{O} / \mathrm{Na}]$ value in the various stellar regions is indicated (it is $\sim 0.6$ dex in the first-generation protostars and $\sim-2$ dex in second-generation stars). (top) During the main sequence, a slow outflowing equatorial disk forms and dominates matter ejection with respect to radiative winds. (middle) At the beginning of central He burning, the composition of the disk material spans the range in $[\mathrm{O} / \mathrm{Na}]$ observed today in low-mass cluster stars. The star has already lost an important fraction of its initial mass. (bottom) Due to heavy mass loss, the star moves away from critical velocity and does not supply its disk anymore. A radiatively driven fast wind takes over before the products of He burning reach the stellar surface.

The proportion of anomalous to normal star observed today depends on at least two quantities: (i) the number of polluter stars and (ii) the dynamical history of the cluster which may lose during its lifetime first- and second-generation stars in different proportions. Decressin et al. (2008, 2009a) estimated these proportions based on dynamical models for GCs. When internal dynamical evolution and dissolution due to tidal forces are accounted for, starting from an initial fraction of anomalous stars of $10 \%$ produces a present-day fraction of about $25 \%$, still too small compared with the observed $70-85 \%$. If gas expulsion by supernovae is accounted for, a much higher fraction is expected to be produced, in better agreement with the observational requirements (Decressin et al. 2010). 


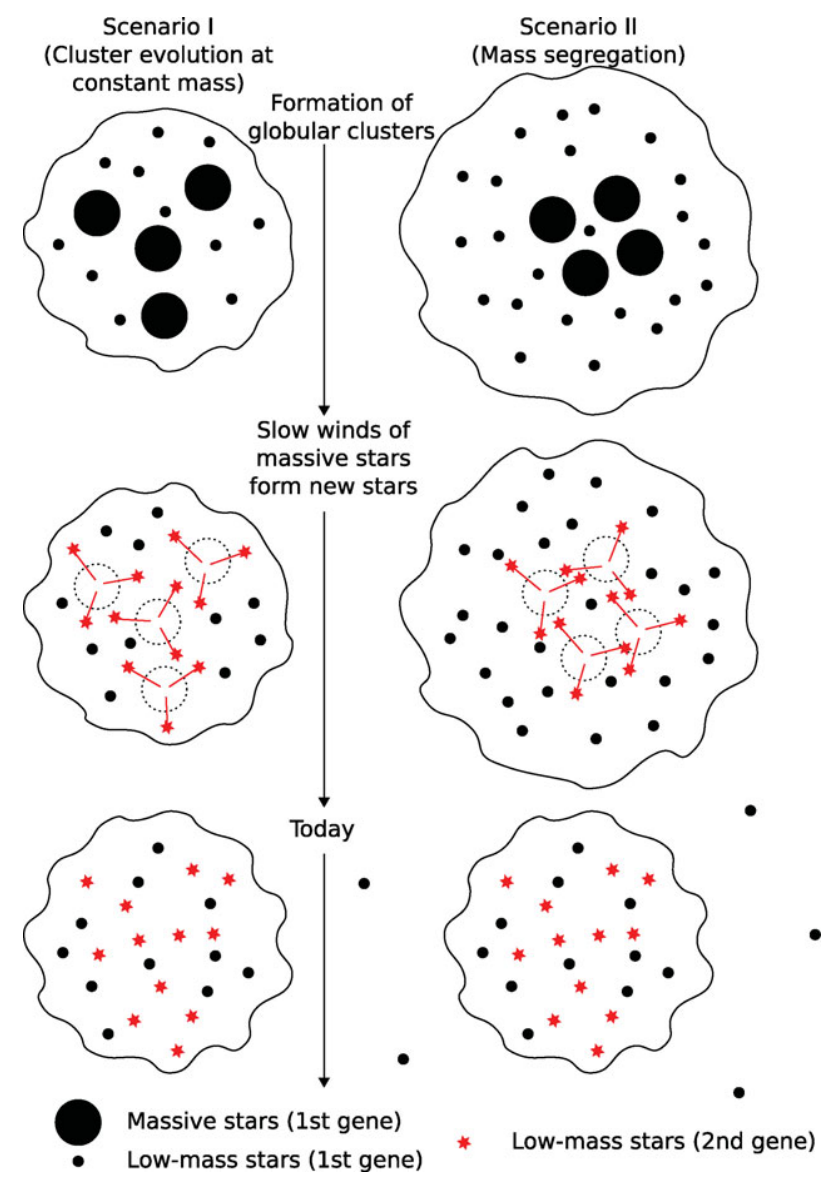

Figure 6. Schematic evolution of a globular cluster: a first generation of stars is formed from a giant molecular cloud. Massive first-generation stars evolve and give birth to a second generation of low-mass stars (dashed symbols in middle panels). Subsequently, the cluster evolves and today (lower panels) a mixture of first- and second-generation low-mass stars is present. In the right panel, the cluster is initially mass segregated and massive stars (and hence second-generation stars) are concentrated towards the cluster centre.

\section{Conclusions}

A significant fraction of stars in globular clusters (about 70-85\%) exhibit peculiar chemical patterns with strong abundance variations in light elements, along with constant abundances in heavy elements. C-N, O-Na and $\mathrm{Mg}-\mathrm{Al}$ anticorrelations, observed both in turnoff stars and in bright giants in GCs, call for a self-enrichment component of the intracluster gas. These features are not seen in field stars and appear to be intrinsic properties of GCs, related to cluster formation itself. They involve CNO cycles and $\mathrm{NeNa}$ and $\mathrm{MgAl}$ chains operating at relatively high temperatures.

Today, there is not a unique, neither a well-defined, nor even a fully consistent scenario for the self-enrichement of GCs. The most commonly invoked scenario assumes that the H-processed ejecta of massive AGB stars of a first generation form a second stellar generation. This idea, however, suffers from several drawbacks from the nucleosynthetic point of view, but also as far as mass budget and IMF of the polluters are concerned.

On the other hand, these abundance anomalies can be created in the H-burning core of a first generation of fast-rotating massive stars and the corresponding elements are 
conveyed to the stellar surface thanks to rotation-induced mixing. If the rotation of the stars is fast enough, this matter is ejected at low velocity through a mechanical wind at the equator. It then pollutes the interstellar medium from which a second generation of chemically anomalous stars can be formed. It is important to note that the "winds of fast-rotating massive stars' scenario to explain the GC chemical properties is only one aspect, among many others, of the evolution of fast-rotating massive stars. For example, that kind of star might also be at the origin of the carbon-rich ultra-metal-poor stars or of the high helium abundance in a subpopulation of $\omega$ Cen.

\section{Acknowledgements}

I would like to thank my main collaborators on the 'Globular Cluster project': Thibaut Decressin, Georges Meynet and Nikos Prantzos. I acknowledge financial support from the IAU, from the French 'Programme National de Physique Stellaire' (CNRS/INSU) and from the Swiss National Science Foundation.

\section{References}

Arnould, M., Goriely, S., \& Jorissen, A. 1999, A\& A, 347, 572

Bonifacio, P., Pasquini, L., Molaro, P., Carretta, E., François, P., Gratton, R. G., James, G., Sbordone, L., Spite, F., \& Zoccali, M. 2007, A\&A, 470, 153

Carretta E., Gratton, R. G., Lucatello, S., Bragaglia A., \& Bonifacio, P. 2005, A $\& A$ A, 441, 131

Carretta, E., Bragaglia, A., Gratton, R. G., Leone, F., Recio-Blanco, A., \& Lucatello, S. 2006, $A \mathscr{S} A, 450,523$

Carretta, E., Bragaglia, A., Gratton, R. G., Lucatello, S., \& Momany, Y. 2007, A\& A, 464, 927

Carretta, E., Bragaglia, A., Gratton, R., D’Orazi, V., \& Lucatello, S. 2009a, $A \& A$, in press

Carretta, E., Bragaglia, A., Gratton, R., \& Lucatello, S. 2009b, A\&SA, 505, 139

Charbonnel, C. 2007, in: F. Kerschbaum, C. Charbonnel \& R. F. Wing, Why Galaxies Care About AGB Stars: Their Importance as Actors and Probes, ASP Conf. Ser., Vol. 378, p. 416, San Francisco: ASP

Chiappini, C., Ekström, S., Meynet, G., Hirschi, R., Maeder, A., \& Charbonnel, C. 2008, A\&A (Letters), 479, L9

Cohen, J. G. 1978, ApJ, 223, 487

Decressin, T., 2007, PhD Thesis, Geneva University

Decressin, T., Baumgardt, H., \& Kroupa, P. 2008, A\&A A, 492, 101

Decressin, T., Baumgardt, H., Kroupa, P., Meynet, G., \& Charbonnel, C. 2009a, in: E. E. Mamajek, D. R. Soderblom, \& R. F. G. Wyse, The Ages of Stars, Proc. IAU Symp. No. 258 , p. 265

Decressin, T., Charbonnel, C., \& Meynet, G. 2007a, A\& A, 475, 859 (DCM07)

Decressin, T., Meynet, G., Charbonnel, C., Prantzos, N., \& Ekström, S. 2007b, A\&̋A, 464, 1029 (DMCPE07)

Decressin, T., Charbonnel, C., Siess, L., Palacios, A., Meynet, G., \& Georgy, C. 2009a, A\&A, 505,727

Decressin, T., Baumgardt, Kroupa, P., Meynet, G., \& Charbonnel, C. 2009b, in: E. E. Mamajek, D. R. Soderblom, \& R. F. G. Wyse, The Ages of Stars, Proc. IAU Symp. No. 258, p. 265

Decressin, T., Baumgardt, Charbonnel, C., \& Kroupa, P., in prep.

Denissenkov, P. A. \& Denissenkova S. N. 1990, SvA Lett., 16, 275

Drake, J. J., Smith, V. V., \& Suntzeff, N. B. 1992, ApJ (Letters), 395, L95

Ekström, S., Meynet, G., Maeder, A., \& Barblan, F. 2008, A\&A, 478, 467

Gratton, R., Sneden, C., Carretta, E., \& Bragaglia, A. 2000, A\&A, 354, 169

Gratton, R., Sneden, C., \& \& Carretta, E. 2004, ARA\&A, 42, 385

Illiadis, C., D'Auria, J. M., Starrfield, S., Thompson, W.J., \& Wiescher, M. 2001, ApJS, 134, 151

Ivans, I. I., Sneden, C., Kraft, R. P., Suntzeff, N. B., Smith, V. V., Langer, G. E., \& Fulbright, J. P. 1999, AJ, 118, 1273 
Langer, G. E., Hoffman, R., \& Sneden, C. 1993, PASP, 105, 301

Langer, G. E. \& Hoffman, R., 1995 PASP, 107, 1177

Lind, K., Primas, F., Charbonnel, C., Grindahl, F., \& Asplund, M. 2009, A\& $A$, 503, 545

Maeder, A. \& Meynet, G. 2000, ARA $\& A, 38,143$

Maeder, A. \& Meynet, G. 2006, A\&A (Letters), 448, L37

Norris, J. E., Cottrell, P. L., Freeman, K. C., \& Da Costa, G. S. 1981, ApJ, 244, 205

Norris, J. E. 2004, ApJ (Letters), 612, L25

Osborn, W. 1971, Observatory, 91, 223

Pasquini, L., Bonifacio, P., Molaro, P, Francois, P, Spite, F., Gratton, R. G., Carretta, E., \& Wolff, B. 2005, A\& $A, 441,549$

Peterson, R. C. 1980, ApJ (Letters), 237, L87

Pilachowski, C. A. 1989, in: G. Cayrel de Strobel, M. Spite \& T. L. Evans (eds.), The abundance spread within globular clusters, Proc. IAU JCM5, p. 1

Prantzos, N. \& Charbonnel, C. 2006, A\&A, 458, 135 (PC06)

Prantzos, N., Charbonnel, C., \& Iliadis, C. 2007, A\& $A$, 470, 179 (PCI07)

Ramirez, S. V. \& Cohen, J. G. 2002, AJ, 123, 3277

]Salpeter1955 Salpeter, E. E. 1955, ApJ, 121, 161

Shetrone, M. D. 1996, AJ, 112, 1517

Sneden, C. 2005, in: V. Hill, P. François \& F. Primas (eds.), From lithium to uranium: Element tracers of early cosmic evolution, Proc. IAU Symp. No. 228, p. 337

Sneden, C., Gratton, R. G., \& Crocker, D. A. 1991, A\&A, 246, 354

Spite, M., Cayrel, R., \& Plez, B. 2005, A\&A A, 430, 655

Ventura, P., D’Antona, F., Mazzitelli, I., \& Gratton, R. 2001, ApJ (Letters), 550, L65

Ventura, P. \& D'Antona, F. 2005a, A\& A, 431, 279

Ventura, P. \& D'Antona, F. 2008a, A\&A A, 479, 805

Yong, D., Grundahl, F., Lambert, D. L., Nissen, P. E., \& Shetrone, M. D. 2003, A\& A, 402, 984

Yong, D., Grundahl, F., Nissen, P. E., Jensen, H. R., \& Lambert, D. L. 2005, A\& A, 438, 875

Yong, D., Aoki, W., \& Lambert, D. L. 2006, ApJ, 638, 1018

Yong, D., Grundahl, F., D’Antona, F., Karakas, A. I., Lattanzio, J. C., \& Norris, J. E. 2009, ApJ (Letters), 695, L62 